\title{
Media-Specific Rate Allocation in Heterogeneous Wireless Networks
}

\author{
Dan Jurca and Pascal Frossard \\ Ecole Polytechnique Fédérale de Lausanne (EPFL) \\ Signal Processing Institute \\ CH-1015 Lausanne, Switzerland \\ Email: $\{$ dan.jurca,pascal.frossard $\}$ epfl.ch
}

\begin{abstract}
We address the problem of joint path selection and rate allocation in multipath wireless streaming, in order to optimize a media specific quality of service. We leverage on the existence of multiple parallel wireless services, in order to enhance the received video quality at a wireless client. An optimization problem is proposed, which aims at minimizing a video distortion metric based on sequence-dependent parameters, and transmission channel characteristics, for a given wireless network infrastructure. Even if joint optimal path selection and rate allocation is in general an NP complete problem, an indepth analysis of the media distortion evolution allows to define a low complexity optimal streaming strategy, under reasonable network assumptions. In particular, we show that a greedy allocation of rates along paths with increasing error probability leads to an optimal solution. We argue that a network path shall not be chosen for transmission, unless all other available paths with lower error probability have been chosen. Moreover, the chosen paths should be used at their maximum end-to-end bandwidth. These results are demonstrated for both independent network paths, and non-disjoint channel segments, in generic network topologies. Simulation results show that the optimal rate allocation carefully trades off total encoding/transmission rate, with the end-to-end transmission error probability and the number of chosen paths. In many cases, the optimal rate allocation provides more than $20 \%$ improvement in received video quality, compared to heuristic-based algorithms.
\end{abstract}

\section{INTRODUCTION}

With the development of novel wireless technologies and increasing available bandwidth, multimedia applications over wireless networks become attractive for both businesses and end users. Fast deployment of Wi-Fi HotSpots, increase in wireless coverage of remote habitable areas (Wi-Max, or wireless mesh networks), improved data services over the $2 \mathrm{G}$ cellular systems and the long awaited debut of $3 \mathrm{G}$ wireless services offer as many potential and inter-operable communication solutions. Recent commercial products [1] offer transparent data services by opportunistically switching the packet routing among multiple wireless services like UMTS, GPRS and WiFi. Service switching is performed by simultaneously probing all available wireless services and by routing data through the service that offers the best channel at a given time. Different research [2] proposes channel switching techniques in ad-hoc wireless networks with the final goal of increasing the overall capacity and the maximum number of supported data flows. It is only a question of time until commercial products will be able to aggregate at one client the simultaneous performance of several available wireless services.

However, the viability of a streaming application over heterogeneous wireless networks mostly depends on the ability to meet stringent QoS requirements, especially in terms of low transmission error, and sustained streaming rate. As the wireless services are still far from providing any widely deployed guarantee of service solution, efficient media streaming strategies have to be devised to get the best out of the available network infrastructure. Lately, multipath streaming emerged as a valid solution to overcome some of the lossy network path limitations [3], [4]. It allows for an increase in streaming bandwidth, by balancing the load over multiple network paths between the media server and the client. It also provides means to limit packet loss effects, when combined with error resilient streaming strategies, and scalable encoding capabilities of the latest encoding standards [5]-[8].

Most of the scientific work dedicated to multi-path streaming focuses on the coding or scheduling processes, but generally not towards finding which paths should ideally be used for the streaming application. Most of these works rely on classic routing algorithms that find the best path (or set of paths) given some established network metrics. While this may be optimal in terms of network utilization, it is certainly suboptimal from a media streaming application viewpoint. In $30-80 \%$ of the cases, the best paths found by classic routing algorithms are suboptimal from a media perspective [9].

Our work proposes to address the problem of streaming path allocation, which takes into account media aware metrics during the decision process. The early work in [10] derives a few empirical rules on what paths should be considered by the streaming application, based on experimental data. These rules consider network metrics (e.g., available bandwidth, loss rate and hop distance), and other media aware metrics (e.g., link jointness/disjointness, video distortion). We provide a more general framework for the analysis of joint path and rate allocation in multipath streaming, driven by media-specific metrics. We consider a network model composed of multiple flows, and a streaming server that can adapt the media source rate to the transmission conditions (by scalable coding, or transcoding, for example). A generic video distortion metric is proposed, which encompasses both the source distortion (mostly driven be the encoding rate), and the channel distortion, dependent on the packet loss probability. 


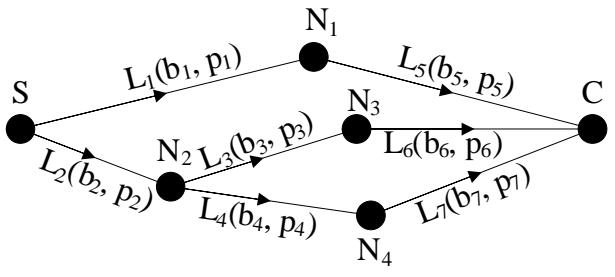

Fig. 1. Large Scale Network with Overlay.

The optimal rate allocation problem is in general an NP complete problem in generic network scenarios. However, a careful analysis of the video distortion evolution under common wireless network assumptions, allows to derive a linear complexity algorithm for the joint optimal path selection, and rate allocation. In other words, our main objective is to jointly find (i) the optimal encoding or streaming rate of a video stream so that the quality at receiver is maximized, and (ii) which network paths should be used for relaying the video stream to the client. Interestingly enough, our conclusions show that the answer to these two questions is represented by a careful tradeoff among available network bandwidth (translated into video encoding rate), transmission loss process, and number of utilized paths. And, in contrary to the commonly admitted opinion, flooding the network in using all the possible paths rarely provides an efficient strategy.

The main contributions of this paper can be briefly summarized as follows:

- We propose a general framework for media streaming analysis, which encompasses network and media aware metrics;

- We perform the first theoretical analysis on the optimality of number, and selection of network paths during the streaming process;

- We provide a linear time media aware routing algorithm that outputs the optimal set of network paths to be used in the streaming process.

The paper is organized as follows: Section II presents the streaming framework and formulates our optimization problem. The theoretical analysis of the streaming process is developed in Section III. Section IV presents the routing algorithm and Section V presents our main results. We present the related work in Section VI, and conclude the paper in Section VII.

\section{Distortion Optimized Multipath Media STREAMING}

\section{A. Multipath Network Model}

We consider a generic heterogeneous network infrastructure, where multiple paths are available between the media server and the client. A typical example of such a topology is represented by a wireless network, or an hybrid topology, where a wireless client accesses the media server through wireless access points, connected to the media server via high rate links. The wireless client $C$ can aggregate the received information on multiple paths, simultaneously benefitting from multiple wireless services. The media packets can be forwarded to the wireless client with the help of base stations (in the case of cellular wireless services), wireless access points (in the case of wireless hot-spots), and/or other wireless nodes (in the case of ad-hoc networks).

The available network between the server $S$ and the client $C$ is modelled as a graph $G(V, E)$, where $V=\left\{N_{i}\right\}$ is the set of forwarding nodes in the network, and $E$ is the set of links or segments (see Figure 1). Each wireless link $L_{u}=\left(N_{i}, N_{j}\right) \in$ $E$ connecting nodes $N_{i}$ and $N_{j}$ has two associated positive metrics:

- the available wireless channel capacity or bandwidth allocated to the streaming application, $b_{u}>0$ expressed in some appropriate unit (e.g., kbps), and,

- the average packet loss probability on the wireless channel $p_{u} \in[0,1]$, related to an iid packet loss process, assumed to be independent of the streaming rate. ${ }^{1}$

Let $\mathcal{P}=\left\{P_{1}, \ldots, P_{n}\right\}$ denote the set of available loop-free paths between the server $S$ and the client $C$ in $G$, with $n$ the total number of non-identical end-to-end paths. A path $P_{i}=\left(S, N_{i}, N_{j}, \ldots, C\right)$ is defined as an ordered list of nodes and their connecting links, such that, no node appears more than once, and that each link $L_{u}$ between two consecutive nodes in the path belongs to the set of segments $E$. Let further $b_{i}$ and $p_{i}$ denote respectively the end-to-end bandwidth and loss probability of path $P_{i}$. We define the bandwidth of an individual path $P_{i}$ as the minimum of the bandwidths among all links on the path (i.e., the "bottleneck bandwidth"). Hence, we have

$$
b_{i}=\min _{L_{u} \in P_{i}}\left(b_{u}\right) \text {. }
$$

Under the commonly accepted assumption that the loss process is independent on two consecutive segments, the endto-end loss probability on path $P_{i}$ becomes a multiplicative function of the individual loss probabilities of all segments composing the path. It can be written as:

$$
p_{i}=1-\prod_{L_{u} \in P_{i}}\left(1-p_{u}\right) .
$$

Please notice that our underlying assumption is that multiple wireless channels, that connect the same wireless client, can be used simultaneously in an independent fashion (e.g., the available rate and loss process of one path is not influenced by the use of another path). This is true in the case of aggregating wireless services working in different spectrum ranges (e.g. UMTS, GPRS and IEEE 802.11), or when the frequency of the wireless channels used are well separated (e.g. 4 independent channels out of 13 in IEEE 802.11 in Europe and 3 out of 11 in USA). Our work can however be slightly modified to take into account the cross-influence of multiple wireless channels to the same wireless client, as discussed later in this paper.

\footnotetext{
${ }^{1}$ Note that the available rate and loss probability of any network segment can be predicted in realtime by any network estimation mechanism [11]
} 
In this network model, efficient streaming strategies have to carefully allocate the rate between the different network paths. The goal of the next sections is to get the best out of the multipath network from a media-driven quality of service perspective.

\section{B. Media-Driven Quality of Service}

The end-to-end distortion, as perceived by the media client, can generally be computed as the sum of the source distortion, and the channel distortion. In other words, the quality depends on both the distortion due to a lossy encoding of the media information, and the distortion due to losses experienced in the network. The source distortion $D_{S}$ is mostly driven by the encoding or streaming rate $R$, and the media sequence content, whose characteristics influence the performance of the encoder (e.g., for the same bit rate, the more complex the sequence, the lower the quality). The source distortion decays with increasing encoding rate; the decay is quite steep for low bit rate values, but it becomes very slow at high bit rate. The channel distortion $D_{L}$ is dependent on the loss probability $\pi$, and the sequence characteristics. It is roughly proportional to the number of video entities (e.g., frames) that cannot be decoded. The end-to-end distortion can thus be written as:

$$
D=D_{S}+D_{L}=f(R, \pi, \Gamma),
$$

where $\Gamma$ represents the set of parameters that describe the media sequence. In low to medium bit rate video streaming, appropriate for wireless transmissions, a commonly accepted model for the source rate distortion is a decaying exponential function on the encoding rate, while the channel distortion is proportional to the number of lost packets (i.e., the packet loss probability, when the number of packet per frame is independent of the bit rate) [12]. Hence, we can explicitly formulate the distortion metric as:

$$
D=\alpha \cdot R^{\xi}+\beta \cdot \pi
$$

where $\alpha, \beta \in \Re^{+}$and $\xi \in[-1,0]$ are parameters that depend on the video sequence, $\Gamma$. This distortion model is a simple and general approximation that follows closely the behavior of more sophisticated distortion measures, such as those proposed in [13], [14]. Since it is suitable for most common streaming strategies where the number of packet per frame is independent of the encoding rate, we use the model of Eq. (4) in the remainder of that paper.

The total streaming rate $R$, and the end-to-end loss probability $\pi$ directly depend on the path selection, and the rate allocation. In the multipath scenario described before, the media application uses rate allocation $\vec{R}=\left[r_{1}, \ldots r_{n}\right]$, where the rate $r_{i}$, with $0 \leq r_{i} \leq b_{i}$, represents the streaming rate on path $P_{i} \in \mathcal{P}$. The total media streaming rate $R$ is expressed as:

$$
R=\sum_{i=1}^{n} r_{i} \leq \sum_{i=1}^{n} b_{i}
$$

The packet loss probability $\pi$ experienced by the media application can be computed as the average of the loss probabilities of the $n$ paths:

$$
\pi=\frac{\sum_{i=1}^{n} p_{i} \cdot r_{i}}{\sum_{i=1}^{n} r_{i}} .
$$

Recall however, that the above definition of streaming paths does not guarantee any two paths in $\mathcal{P}$ to be completely disjoint. Therefore, $\vec{R}$ is a valid rate allocation on the network graph $G$, if and only if $G$ can simultaneously accommodate the rates on all paths in $\mathcal{P}$. A necessary condition for the equality in the right side of Eq. (5) to be verified requires therefore that all bottleneck links of the $n$ streaming paths are disjoint. Sufficient conditions for valid rate allocation are analyzed in the next section.

\section{From Network Graph to Flow Tree}

In order to study multipath rate allocation in the overlay network, we first propose to represent the network graph $G$ as a flow tree. The media server becomes the root of the tree, and each flow $\mathcal{F}_{i}$ represents the share of the overall media stream, which is sent on a network path $P_{i}$. The media stream is the composition of individual media flows, and the client is represented as a set of leaf nodes, with one leaf per flow. The rate allocation therefore becomes a flow assignment problem.

Considering that there is (at most) one flow for each network path $P_{i}$, we can transform the original network graph $G$ into a flow tree by duplicating any network edge and vertex that is shared by more than one network path, as represented in Figure 2. Since the transformation from paths to flows is bijective, each flow is characterized by a maximal end-to-end streaming rate, and an end-to-end loss probability, as computed in Section II-A. The flow $\mathcal{F}_{i}$ on path $P_{i}$ is using a streaming rate $r_{i} \leq b_{i}$ with a loss probability $p_{i}$.

Due to the assumption of rate independent loss process, any two flows in the tree are independent in terms of loss probability. However, flows may be dependent in terms of aggregated bandwidth, since they may share joint bottleneck links. The flow tree representation allows us to explicit the constraints imposed on a valid rate allocation. These constraints are imposed by bandwidth limitation on the network links, and flow conservation in the network nodes. The necessary and sufficient conditions for the flow tree model to be a valid representation of the original network graph can finally be grouped into single flow, and multiple flow constraints, and expressed as:

1) Single Flow Constraints:

- path bandwidth limitations: $r_{i} \leq b_{i}, \forall P_{i} \in \mathcal{P}$;

- flow conservation at intermediate nodes: for every node $N_{j} \in P_{i}, r_{i}^{i n}=r_{i}^{\text {out }}=r_{i}$, where $r_{i}^{\text {in }}$ and $r_{i}^{\text {out }}$ are the incoming and respectively outgoing rates of $\mathcal{F}_{i}$ passing through node $N_{j}$.

2) Multiple Flow Constraints:

- link bandwidth limitations:

$\sum_{P_{i}: L_{u} \in P_{i}} r_{i} \leq b_{u}, \forall L_{u} \in E$; 


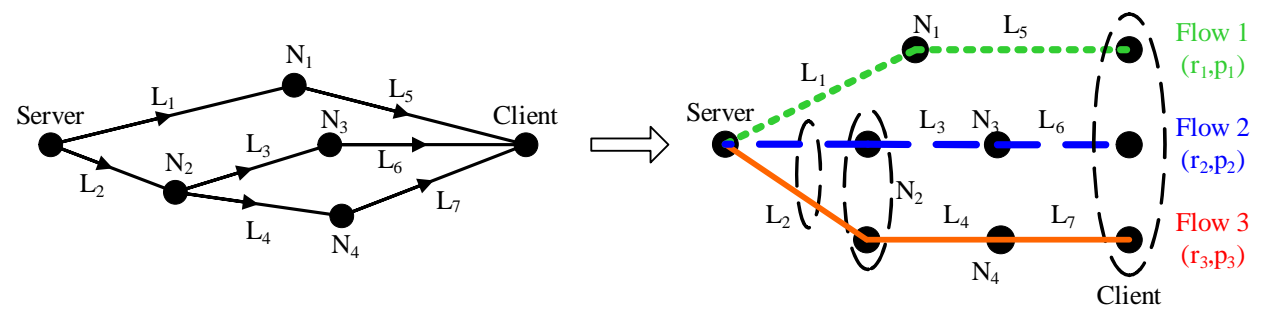

Fig. 2. Equivalent transformation between a network graph and a tree of paths between the server and the client.

- flow conservation at intermediate nodes: for every node $N_{j} \in V$ : $\sum_{P_{i}} r_{i}^{\text {in }}=\sum_{P_{i}} r_{i}^{\text {out }}=\sum_{P_{i}} r_{i}, \forall P_{i}: N_{j} \in P_{i}$.

\section{Multipath Rate Allocation: Problem Formulation}

Now that the network model and rate constraints have been presented, we can formulate the optimized multipath rate allocation problem as follows. Given the network graph $G$, the optimization problem consists in jointly finding the optimal streaming rate for the video sequence, along with the optimal subset of network paths to be used for transmission, such that the end-to-end distortion is minimized.

Equivalently, using the flow tree representation of the network graph proposed in Section II-C, the optimization problem translates into finding the optimal rate allocation for each of the flows in the tree, such that the video distortion is minimize. It can be enounced as follows:

Multimedia Rate Allocation Problem (MMR): Given the network graph $G$, the number of different paths or flows $n$ and the video sequence characteristics $(\Gamma=(\alpha, \beta, \xi))$, find the optimal rate allocation $\overrightarrow{R^{*}}=\left[r_{1}, \ldots r_{n}\right]^{*}$ that minimizes the distortion metric $D$ :

$$
\begin{aligned}
\overrightarrow{R^{*}} & =\underset{\vec{R}}{\arg \min } D\left(r_{1}, \ldots r_{n}\right) \\
& =\underset{\vec{R}}{\arg \min }\left(\alpha \cdot R^{\xi}+\beta \cdot \pi\right)
\end{aligned}
$$

where $R=\sum_{i=1}^{n} r_{i}$ and $\pi=\frac{\sum_{i=1}^{n} p_{i} \cdot r_{i}}{\sum_{i=1}^{n} r_{i}}$, under (i) Single Flow Constraints and (ii) Multiple Flow Constraints, as defined above.

The solution of the optimization problem by integration of the constraints into a Lagrangian formulation is not straightforward, mainly because of the non-convexity of the optimization function, and of the numerous multiple flow constraints. However, in the next section, we present a careful study of the distortion metric that leads to the definition of three main theorems, used to derived a low complexity rate allocation strategy. They show that it is always best to use first the network paths with the lowest loss probability. In the same time, they show that there is a tradeoff between encoding source rate (equivalent to the transmission rate in our scenario), and the loss process that affects the transmission. A short intuition is provided for each of the theorems (please refer to [15] for detailed proofs).

\section{Optimal Flow Rate Allocation}

\section{A. Illustrative Example}

Let us first take a simple example to illustrate the behavior of the end-to-end video distortion in a multipath scenario. We consider a basic network scenario consisting of two disjoint network paths, $P_{1}$ and $P_{2}$, with bandwidth $b_{1}=b_{2}=$ $1000 \mathrm{kbps}$, and loss probabilities $p_{1}=2 \%$ and $p_{2}=4 \%$, respectively. Consider two independent streams $\mathcal{F}_{1}$ and $\mathcal{F}_{2}$, traversing the two network paths with streaming rates $r_{1} \leq b_{1}$, and $r_{2} \leq b_{2}$. The evolution of the distortion function given in Eq. (4) is presented in Figure 3, for a test video sequence.

As expected, we observe that the decrease in distortion is larger if we increase the rate of flow $\mathcal{F}_{1}$, than if we equivalently increase the rate of flow $\mathcal{F}_{2}$. This behavior is due to the lower loss probability that affects the path followed by the flow $\mathcal{F}_{1}$. In the same time, we observe that the distortion metric is always decreasing with the increase of $r_{1}$, hence it is optimal to fully utilize the bandwidth of the path with the smallest loss probability.

More interestingly, Figure 4 shows that the behavior of the distortion as a function of the rate $r_{2}$, depends on the value of the rate $r_{1}$. For high values of $r_{1}$, the distortion can even increase with growing rate $r_{2}$. In other words, beyond a given value of the streaming rate on the most reliable network path, adding an extra flow can degrade the end-to-end quality of the media application. In this case, the negative influence of the error process on the second network path is greater than the improvement brought by additional streaming rate. Such a behavior is the key to explain why using all the paths to their full bandwidth does not necessarily result in an efficient streaming strategy.

\section{B. Independent Paths}

We now generalize the previous observations, and derive theorems that guide the design of an optimal rate allocation strategy. This section shows that, in the optimal rate allocation between independent paths, a flow is either used at its full bandwidth, or not used at all. Furthermore, the optimal rate allocation always chooses the lowest loss probability paths, i.e., a path cannot be selected, unless all other paths with a lower loss probability have been picked before. We start from an ideal streaming scenario with fully disjoint network paths, 


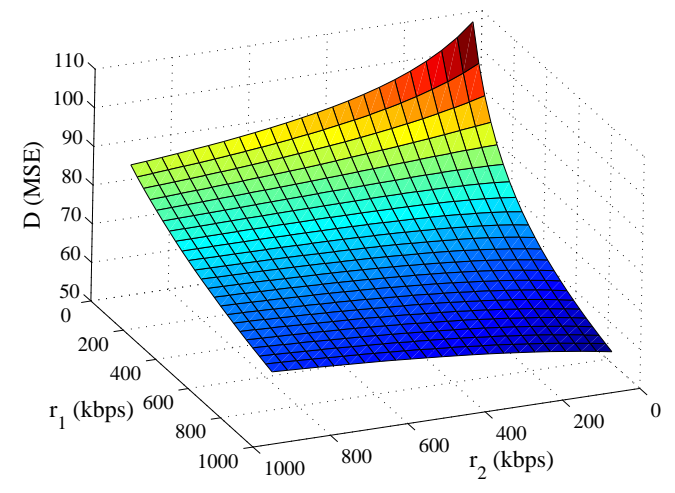

Fig. 3. Distortion measure for two network paths in function of available rates, $\alpha=1.76 \cdot 10^{5}, \xi=-0.658, \beta=1750$, $p_{1}=0.02, p_{2}=0.04$.

and eventually add flow constraints, which are however shown not to affect the initial findings.

Assume that the $n$ disjoint network paths are represented into a tree of flows as explained in Section II-C. Without loss of generality, we further assume that flows $\mathcal{F}_{i}$ with $1 \leq i \leq n$, are arranged in increasing order of the loss probability, i.e., $p_{1}<p_{2}<\ldots<p_{n}$. We note that, from the distortion metric point of view, any two flows $\mathcal{F}_{i}$ and $\mathcal{F}_{j}$, traversing paths $P_{i}$ and $P_{j}$ with the same loss probability $p_{i}=p_{j}$, can be observed as a single flow affected by the same loss probability $p_{i}$, and having an aggregated rate $r_{i}+r_{j}$. Under these generic settings, we first claim that the optimal rate allocation either uses a network path to its full bandwidth, or does not use it at all.

Theorem 1 (On-Off Flows): Given a flow tree with independent flows $\mathcal{F}_{i}$ having rates $r_{i} \in\left[0, b_{i}\right]$ and a distortion metric as defined in Eq. (4), the optimal solution of the MMR problem when all the paths are disjoint, lies at the margins of the value intervals for all $r_{i}$, i.e., the optimal value of $r_{i}$ is either 0 or $b_{i}, \forall i: 1 \leq i \leq n$.

Corollary 1: Given a flow tree with independent flows $\mathcal{F}_{i}$ having rates $r_{i} \in\left[0, b_{i}\right]$ and a distortion metric as defined in Eq. (4), the optimal solution of the MMR problem when all paths are disjoint, allocates $r_{1}=b_{1}$, where the path $P_{1}$ is the path with the lowest loss probability.

Intuitively, the previous theorem can be justified by the fact that the total media distortion introduced by the losses on a given network path does not depend on the transmission rate on that path. Hence, since the source distortion decreases with increased transmission rate, it is always best to fully utilize the capacity of a transmission path.

Theorem 1 greatly reduces the search space for an optimal solution for the MMR optimization problem. Hence we can rewrite the optimal streaming solution as a vector $\Phi$ of boolean values $\phi_{i}$ for each flow $\mathcal{F}_{i}$, where $\phi_{i}=1$ means that path $P_{i}$ is used with full rate $r_{i}=b_{i}$, and $\phi_{i}=0$ denotes that the path $P_{i}$ is not used by the streaming application. The previous corollary further says that $\Phi=\left[\phi_{1}=1, \phi_{2}, \ldots, \phi_{n}\right]$ is part of the optimal solution.

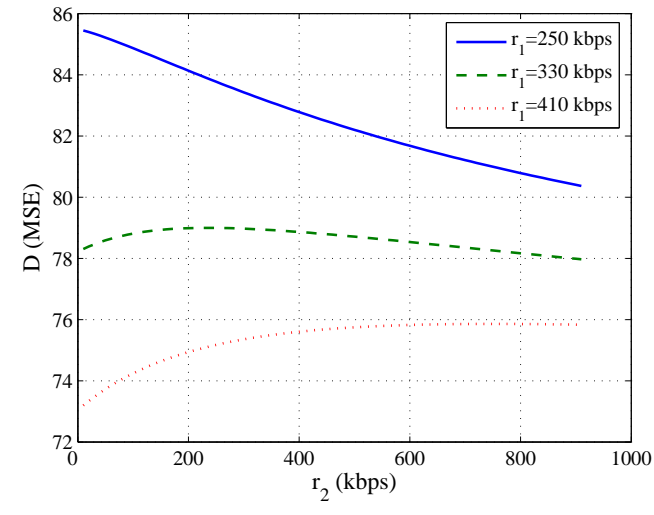

Fig. 4. Distortion behavior as a function of $r_{2}$, for various fixed values of $r_{1}$.

For bounded intervals for all rates $r_{i}, 2^{n-1}$ computations are sufficient for finding the optimal solution vector. For practical scenarios, with a limited number of available network paths, between a server and a client, this number of computations is in general quite low. We can however further constrain the search space by considering that the optimal rate allocation always uses first the network paths with the smallest loss probabilities.

Theorem 2 (Parameter Decoupling): Given a flow tree with independent flows $\mathcal{F}_{i}$ having rates $r_{i} \in\left[0, b_{i}\right]$ and a distortion metric as defined in Eq. (4), the structure of the optimal rate allocation is $\Phi^{*}=[1,1, \ldots, 1,0,0, \ldots 0]$.

Intuitively, this theorem translates into the fact that starting from an initial rate allocation on the available network paths, in terms of distortion you can always obtain a better allocation by transferring transmission rates to paths characterized by lower loss probabilities.

The previous theorems, show that we can find the optimal solution for our optimization problem by iteratively searching all available network paths $P_{i}$, taken in ascending order of their loss probability $p_{i}$. Once we find a network path that can improve the overall distortion result, before using it, we have to make sure that all other network paths with better loss parameters are already used to their maximum available bandwidth. Hence, the search space is reduced to $n$ computations (i.e., the complexity increases linearly with the number of flows).

\section{Non-Disjoint Network Paths}

We now show that, relaxing the assumption on disjoint network paths in the original network graph does not change the general form of the optimal solution. We assume that in the original network graph $G$, there is at least one bottleneck link $L_{u}$, shared by at least two distinct network paths. Let $\mathcal{P}_{u}=\left\{P_{k}\right\}, \forall k: L_{u} \in P_{k}$, be the set of paths sharing the bottleneck link $L_{u}$. In this particular case, while using any of the paths $P_{k}$ alone will yield an available bandwidth $b_{k} \leq b_{u}$, using all of them in the same time will yield an 
aggregated bandwidth $b_{u} \leq \sum_{k} b_{k}$. Note that $L_{u}$ may, or may not be a bottleneck link for any of the paths $P_{k}$, treated independently. Paths $P_{k}$ are called "joint paths". The following theorem regulates the sharing of bandwidth $b_{u}$ among paths $P_{k}$ :

Theorem 3 (Bottleneck Bandwidth Sharing): Let $L_{u}$ be a bottleneck link for the set of paths $\mathcal{P}_{u}=\left\{P_{k}\right\}$ in $G$, the bottleneck link bandwidth $b_{u}$ shall be shared among paths $P_{k}$ in a greedy way, starting with the path affected by the lowest loss probability.

This theorem can be proved by a similar argument to the ones used in the previous ones. Note that Theorem 3 can easily be extended to any number of bottleneck links in $G(V, E)$, and to paths that belong to different sets $\mathcal{P}_{u}$ in the same time. Theorem 3 allows to extend Theorem 2 to generic network graphs, with potentially non-disjoint paths. It results in the general rule that paths should be taken in the increasing order of their loss probability, and that all the flows should be used to their maximum capacity, which can be limited by joint bottleneck links, before considering an additional flow. Interestingly, any network scenario can thus be transformed into a disjoint flow tree, by a greedy allocation of joint bottleneck bandwidths to flows affected by lower loss probabilities first. After this transformation, applying Theorem 1 and Theorem 2 will yield the optimal rate allocation for the given streaming scenario.

We can further relax the assumption of independent flows in Theorem 1, by proper adaptation of the maximal bandwidth of all non-disjoint paths.

Corollary 2: Given a generic flow tree with $\mathcal{F}_{i}$ ordered in increasing order of their loss probability, and having rates $r_{i} \in\left[0, b_{i}\right]$, and a distortion metric as defined in Eq. (4), the optimal solution of the MMR problem lies at the margins of the value intervals for all $r_{i}$, i.e., the optimal value of $r_{i} \forall i: 1 \leq i \leq n$, is either 0 or $b_{i}^{\prime}=\min \left(b_{i}, w_{i}\right)$, where $w_{i}=\min _{u: L_{u} \in P_{i}}\left\{b_{u}-\sum_{k: L_{u} \in P_{k} \text { and } p_{k}<p_{i}} b_{k}^{\prime}\right\}$.

Interested readers are referred to [15] for detailed proofs of the theorems presented above.

\section{Linear Complexity Rate Allocation ALGORITHM}

This section presents a simple algorithm that computes the optimal rate allocation for the optimization problem. The previous theorems represent the keys for a fast search through the flow tree. Assume that the sever knows, or can predict the parameters of the intermediate network links, and the sequence-dependent distortion parameters. The encoding rate can be adapted at the server by adaptive or scalable encoding, or transcoding. Initially, the network graph is transformed into a tree of flows $\mathcal{F}_{i}$, sorted along increasing values of the loss probabilities $p_{i}$, with greedy assignment of joint bottleneck link bandwidths. In case where two network paths have the same end-to-end loss probability, they are considered as a single path with aggregated bandwidth.
The search for an optimal solution of the shape given by Theorem 2 is performed then iteratively. Indeed, the analysis proposed in Section III shows that a simple algorithm can find the optimal rate allocation by parsing all available network paths in ascending order of their loss probability. Denote $\Phi_{i}=$ $\left[\phi_{1}, \ldots, \phi_{n}\right]$ a solution vector with $\phi_{j}=1, \forall j \leq i$ and $\phi_{j}=0$ otherwise. $R\left(\Phi_{i}\right)=\sum_{j=1}^{i} r_{i}$ becomes the cumulative rate of the first $i$ flows, whose individual rates have been chosen according to Corollary 2 . The overall loss probability of the first $i$ flows, $\pi\left(\Phi_{i}\right)$, is then given by $\pi\left(\Phi_{i}\right)=\frac{\sum_{j=1}^{i} p_{j} \cdot r_{j}}{\sum_{j=1}^{i} r_{j}}$. The Search Algorithm iteratively computes $D\left(R\left(\Phi_{i}\right), \pi\left(\Phi_{i}\right)\right)$, for $1 \leq i \leq n$, and the optimal rate allocation is the policy $\Phi^{*}$ that minimizes the distortion metric:

$$
\Phi^{*}=\underset{\Phi_{i}, 1 \leq i \leq n}{\arg \min } D\left(R\left(\Phi_{i}\right), \pi\left(\Phi_{i}\right)\right)
$$

When the algorithm finishes the search of all flows, it stops and outputs the optimal multipath rate allocation strategy. Algorithm 1 proposes a sketch of the rate allocation algorithm.

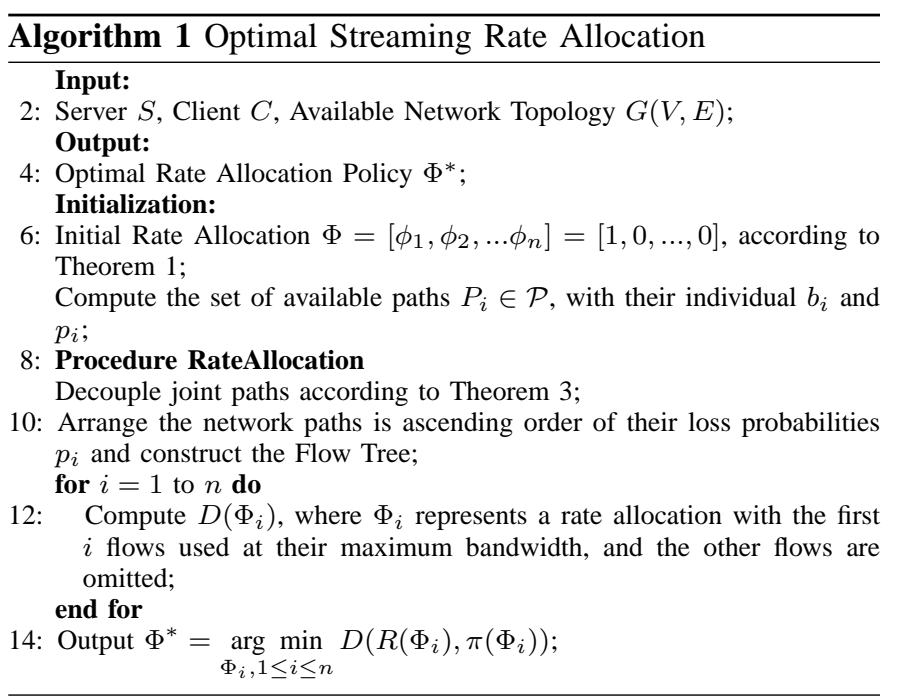

During the initialization process, Algorithm 1 must compute all available paths between the streaming server $S$ and the client $C$. This is a well-known problem in graph theory, and a solution can be easily found by implementing a depth-first search $(D F S)$ [16], for example. The algorithm then arranges the discovered network paths as a flow tree in ascending order of their end-to-end loss probabilities. Any sorting algorithm of complexity $O(n \log (n))$ can be used. After the flow tree is constructed, the core of the algorithm finds the optimal rate allocation with a complexity $O(n)$.

Notice that the possible influences on rate and loss process among different simultaneously used network paths are not taken into account in the presented algorithm. However, on such network scenarios, the locality information for the wireless nodes, along with a model for the influence on the total 


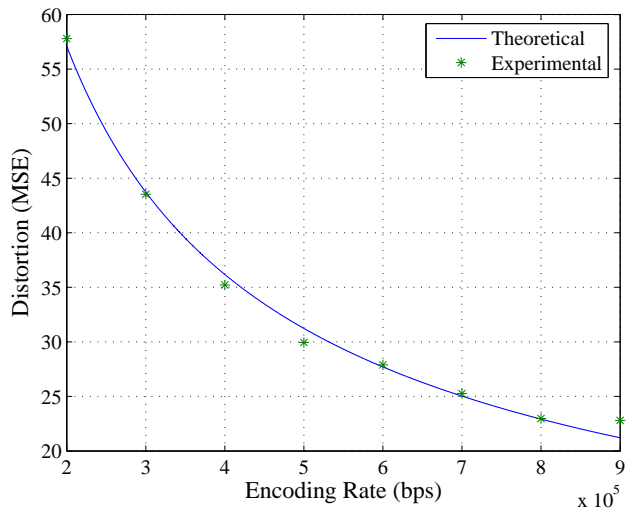

(a) Encoding Rate Distortion Validation

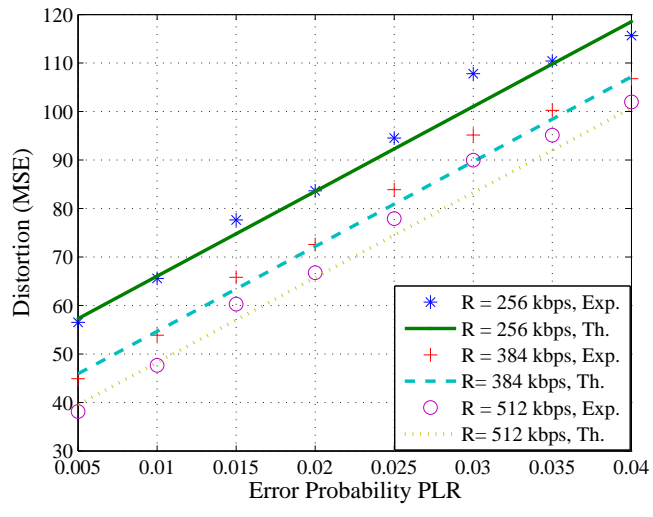

(b) Loss Distortion Validation

Fig. 5. Distortion Model Validation with Video Streaming Experiments using the H264 encoder.

available rate and loss process of multiple used transmission paths, can be easily factored in during the rate allocation decision process.

\section{Simulation Results}

\section{A. Simulation Setup}

We test our optimal rate allocation algorithm in different random wireless network scenarios, and we compare its performance to heuristic rate allocation algorithms. We use an H.264 encoder, and the decoder implements a simple frame repetition error concealment strategy in case of packet loss. We concatenated the foreman_cif sequence to produce a 3000 frame-long video stream, encoded at 30 frames per second. The encoded bitstream is packetized into a sequence of network packets, each packet containing information related to one video frame. The packets are sent through the network on the chosen paths, in a FIFO order, following a simple earliesttransmission-time-first scheduling algorithm. We further consider a typical video-on-demand $(V o D)$ streaming scenario, where the admissible playback delay is large enough (larger than the time to transmit the biggest packet on the lowest bandwidth path). Hence, a video packet is correctly decoded at the client, unless it is lost during transmission due to the errors on the network links.

Our simulations first validate the distortion metric proposed in Eq. (4). Then, the performance of our optimal rate allocation algorithm is compared to heuristic rate allocation algorithms, on a set of random network topologies.

\section{B. Distortion Model Validation}

The video sequence is encoded at rates between $200 \mathrm{kbps}$ and $1 M b p s$, and the mean-square-error $(M S E)$ between the original sequence and the decoded one is computed, in errorfree scenario. Simulation results are compared in Figure 5(a) to the distortion model values, whose parameters have been set to $\alpha=1.7674 \cdot 10^{5}, \xi=-0.65848$, and $\beta=1750$, respectively. We observe that the theoretical distortion curve closely follows the experimental data, which validates the theoretical model for the source distortion part.
TABLE I

PARAMETERS FOR RANDOM GRAPH GENERATION

\begin{tabular}{|c|c|}
\hline Parameter & Wireless Scenario \\
\hline \hline Nr. of Nodes & 10 \\
\hline Connectivity Probability $\gamma$ & 0.6 \\
\hline$R_{\min }$ & $10^{5} \mathrm{bps}$ \\
\hline$R_{\max }$ & $7 \cdot 10^{5} \mathrm{bps}$ \\
\hline$P L R_{\min }$ & $10^{-3}$ \\
\hline$P L R_{\max }$ & $4 \cdot 10^{-2}$ \\
\hline
\end{tabular}

In order to validate the loss distortion component $D_{L}$, random errors are introduced during the network transmission process, where each packet is lost with an independent loss probability $P L R$. Simulations are performed with different values of loss probabilities, and different encoding rates. We observe in Figure 5(b) that the theoretical model closely approximates the experimental data, where each experimental point is averaged over 10 simulation runs. Even if it stays quite simple, the distortion model used in our work closely fits the average behavior of lossy video streaming scenarios. Note that the sequence-dependent parameters may obviously have different values for other encoders or other video sequences. The evolution of the distortion function however stays the same, independently of the exact values of these parameters, which could even be fixed for a given class of video sequences.

\section{Optimal Rate Allocation Algorithm Performance}

We now present the performance of the proposed optimal rate allocation algorithm, in various random wireless network scenarios (Figure 6).

We generate 500 random graphs, where any two nodes are directly connected with a probability $\gamma$. The parameters for each edge are randomly chosen according to a normal distribution, in the interval $\left[R_{\min }, R_{\max }\right]$, for the bandwidth, and respectively $\left[p_{\min }, p_{\max }\right]$ for the loss probability. The parameters for the wireless scenario used in our paper are presented in Table I.

For each of the simulated random topologies, we compute the end-to-end media distortion when rates are optimally 


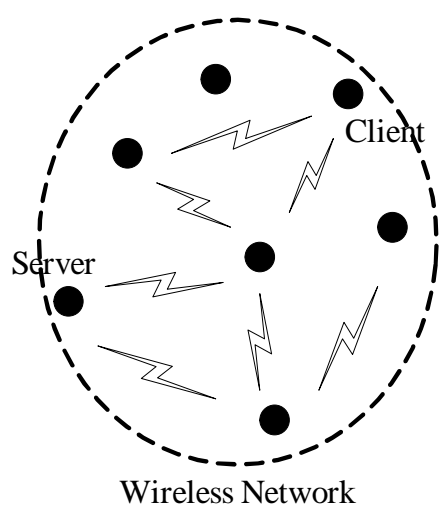

Fig. 6. Random Wireless Network Scenario.

TABLE II

Average Distortion Results ( $M S E$ )

\begin{tabular}{|c|c|c|c|c|c|}
\hline Scenario & $D_{o p t}$ & $D_{P L R}$ & $D_{R}$ & $D_{2 R}$ & $D_{M F}$ \\
\hline \hline Wireless & 91.2 & 99.74 & 122.861 & 143.79 & 108.52 \\
\hline
\end{tabular}

allocated, and we compare it to the results obtained by other simple rate allocation algorithms, namely, (i) a single path transmission scenario, which selects the best path in terms of loss probability, $\left(D_{P L R}\right)$, (ii) a single path transmission scenario, which uses the best path in terms of effective bandwidth or "goodput" computed as $b_{i}\left(1-p_{i}\right),\left(D_{R}\right)$, (iii) a multipath transmission scenario that picks the best two paths in terms of goodput, $\left(D_{2 R}\right)$, and (iv) a multipath transmission scenario that uses the maximum number of available flows, $\left(D_{M F}\right)$. The results, in terms of $M S E$, averaged over 500 random graphs are presented in Table II.

As expected, our algorithm provides the best performance in average over all considered topologies. It has to be noted that, in each individual simulation run, our algorithm never performs worse than any of the heuristic schemes. Also, we observe that the rate allocation that is the closest to the optimal strategy is the one offered by the use of the best single path in terms of loss rate. This can be explained by the high loss probabilities of the intermediate links, which cannot be compensated by extra rate added by subsequent flows. The total average streaming rate over the 500 simulation runs is $R=450 k b p s$.

Next, we study the benefit offered by optimal rate allocation, as compared to the simple heuristic schemes. The relevance of the optimal solution is measured by counting the number of simulation runs in which the optimal rate allocation brings an improvement of $[0-5 \%),[5-10 \%),[10-20 \%)$ and above $20 \%$, in terms of end-to-end video distortion, compared to the other streaming strategies. The results are presented in Figure 7.

As expected, we observe that the best approximation is presented in most of the cases by the lowest loss probability path streaming. Still, in almost $40 \%$ of the simulation runs, the optimal rate allocation improves the distortion result by more than $10 \%$.

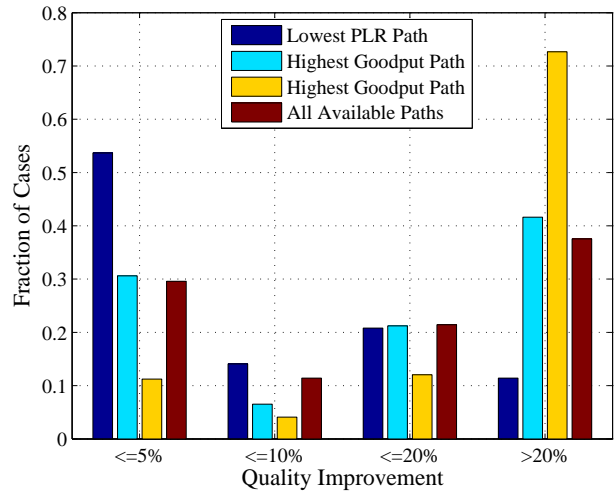

Fig. 7. Wireless Quality

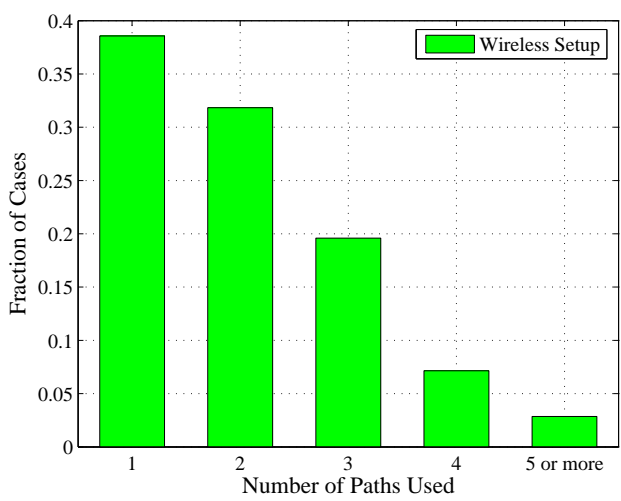

Fig. 8. Distribution of Optimal Number of Paths.

Finally, it is interesting to observe that the rate allocations based on the best goodput path, and best two goodput paths algorithms provide in average the worst results.

We also compute the optimal average number of flows used in each simulation scenario, compared to the average number of available paths. We observe that from an average of available paths of 5.04, the optimal rate allocation uses no more than 2.04 paths.

From the multipath streaming point of view, it interestingly shows that, using a very large number of streaming paths does not contribute to an improvement of the video quality at the receiver. The distribution of the number of flows used per simulation run, is presented in more details in Figure 8.

In summary, we observe that a small number of transmission flows is sufficient for an optimal video quality at the receiver, in all simulation scenarios. Paths with lower error probability should be preferred to higher bandwidth paths in almost all simulated wireless scenarios.

\section{RELATED WORK}

The research community has recently started to investigate the idea of multipath routing and streaming in order to improve the QoS of media applications. The authors of [17] present a distance-vector algorithm for finding multiple paths, while the authors of [18] present a multipath extension of Direct 
Source Routing for wireless ad-hoc environments. The purpose of the algorithms is to achieve load balancing over multiple paths, and to simultaneously minimize delays. The problem of finding disjoint paths in cost networks is further addressed in [19]. The authors formulate an NP complete min-min problem, important for the survivability of a network in case of link failures. Similarly, the authors of [20] formulate in Linear Programming the constrained multipath-routing problem, with the objective of minimizing the maximum link utilization, under multiple constraints. Disadvantages of mutipath routing, in terms of network destabilization, are examined in [21].

While all these works give a detailed analysis of the multipath routing problem from a networking point of view, we rather address the problem from a media application perspective. The process of choosing the paths for transmission and their respective rate allocation is subordinated to achieving a better streaming experience, measured in terms of video distortion. The work presented in [22] addresses a similar problem of choosing the best path from a media perspective. However, the authors only address the question of path switching efficiency from the media application point of view, and do not investigate the benefits of multipath streaming.

More generally, routing with multiple metrics is the target of many works in QoS routing. But QoS routing with multiple constraints is, in general, an NP complete problem. An initial proof, for the case of at least two additive metrics is given in [23]. The authors propose heuristic algorithms for both source routing, and hop-by-hop routing, which find one path satisfying the QoS requirements of multimedia applications. Recent works in multi-constrained routing optimize a linear [24], or non-linear [25] relation between constraints, using low complexity algorithms. A similar function, built on multiple path metrics is used in [26] to find multiple network paths for streaming. Several other works also express the multi-constrained path problem (MCP) in an NP complete formulation, and use fast searching algorithms, e.g., tabusearch, to find a locally optimal solution [27]. In these works, polynomial time algorithms based on heuristics are proposed to provide a general sub-optimal solution to the NP complete QoS routing problem.

In contrary to common QoS routing problems, we propose a media-specific distortion metric, which comprises multiple network link parameters together with media aware parameters. The metric describes the quality of the received video, as a function of the specific network scenario and streaming process. The optimization of the end-to-end distortion translates into choosing the best set of paths, and the respective optimal rate allocation. Classical optimization methods however fail to obtain a simple solution due to the non-convexity of the optimization function. An in-depth analysis of the behavior of this metric however allows to derive a simple algorithm that achieves the optimal solution in linear time.

In parallel, exploiting diversity in wireless ad-hoc or cellular networks has been addressed in [28], [29] and [30]. The main purpose of the works is to reduce routing over-head, to increase the survivability and power efficiency of the network, or to reduce the impact of frequent transmission errors. Even if we also consider here wireless or hybrid streaming scenarios, we are interested in finding the optimal set of paths from an media application perspective. Available network resources are used in order to ensure the best possible transmission quality in terms of received video.

Flow assignment problems have been addressed in [31] and [32]. The authors of the first paper are concerned with optimally splitting the data on multiple disjoint paths in order to avoid packet re-sequencing at the client. The second paper presents an algorithm that minimizes the end-to-end delay of data transmission while complying with an aggregated bandwidth constraint. The algorithm is optimal only in the case of unit capacity links and disjoint paths. Our flow problem formulation is general and deals with both joint and disjoint paths. We show that, from the media application point of view, an optimal flow allocation is achievable in any network scenario, by joint optimization of the number of paths used, and the aggregated rate of the flows.

Finally, the multipath problem is specifically addressed in the case of media streaming in [33]. The authors present a FEC scheme combined with server diversity and a packet scheduling mechanism, which intends to minimize the cumulative distortion of individual erroneous video packets. Our work focuses on a non-multicast communication scenario, with an intermediate network comprising multiple available transmission paths. Multi-stream coding, combined with multipath transmission, has been presented in [34] as a solution to fight against network errors in an ad-hoc network environment. In the same time, the authors of [35] analyze a multiple path streaming scenario for the transmission of a video sequences encoded in multiple descriptions. They minimize an additive distortion metric, computed as the sum of the individual distortions of each of the independent descriptions. For complexity reasons, their analysis is reduced to a scenario comprising two encoded descriptions and two transmission paths. In our work we rather address the questions of how many transmission paths to use, and how to chose them, in order to maximize the efficiency of the streaming application. Our streaming framework is more general, and applicable to any streaming scenario that obeys an additive rule for the aggregated transmitted rate and loss process. The proposed algorithm finds the optimal transmission strategy and encoding rate, based just on the available network topology, and video sequence dependent parameters.

\section{CONCLUSIONS}

In this paper, we propose to use a flow model to analyze the opportunity of multipath media streaming over wireless networks. Based on an equivalent transformation between the available network graph and a tree of flows, we jointly determine the network paths, and the optimal rate allocation for generic streaming scenarios. A media specific performance metric is used, which takes into account the end-to-end network path parameters along with media aware parameters. 
An in-depth analysis of the end-to-end distortion behavior, under specific wireless network assumptions, drives the design of a linear time algorithm for optimal rate allocation, which is in general an NP complete problem. The form of the optimal rate allocation solution follows a simple greedy rule that always uses the paths with the lowest loss probability first. In particular, we show that extra network paths are either used at their maximum available bandwidth, if their value is large enough, or simply ignored. The overall rate allocation solution offers a careful trade-off between extra transmission rate and increase in the end-to-end error process. Even for large network scenarios, only a small number of paths should optimally be used for transmission, taken from the lowest loss probability channels.

The optimal rate allocation algorithm has been tested in various random network scenarios, and it significantly outperforms simpler schemes based on heuristic rate allocation strategies. In many cases, our algorithm even provides an end-to-end distortion improvement of more than 20\%. Due to its low complexity, and important benefits in most streaming scenarios, the optimal rate allocation algorithm provides a very interesting solution to efficient media streaming over resourceconstrained networks.

\section{REFERENCES}

[1] Swisscom Mobile Unlimited UMTS/GPRS/WLAN, http://www.swisscom-mobile.ch/scm/gek_mobile-unlimited-en.aspx.

[2] P. Bahl, R. Chandra, and J. Dunagan, "Ssch: Slotted seeded channel hopping for capacity improvement in ieee 802.11 ad-hoc wireless networks," in Proceedings of ACM Mobicom, October 2004, pp. 216230.

[3] L. Golubchik, J. Lui, T. Tung, A. Chow, and W. Lee, "Multi-path continuous media streaming: What are the benefits?" ACM Journal of Performance Evaluation, vol. 49, no. 1-4, pp. 429-449, Sept 2002.

[4] Y. Li, S. Mao, and S. S. Panwar, "The case for multimedia transport over wireless ad hoc networks," in Proceedings of IEEE/ACM BroadNets, October 2004

[5] T. Nguyen and A. Zakhor, "Distributed video streaming with forward error correction," in Proceedings of the Packet Video Workshop, Pittsburg, PA, 2002.

[6] J. G. Apostolopoulos and M. D. Trott, "Path diversity for enhanced media streaming," IEEE Communications Magazine, vol. 42, no. 8, pp. 80-87, August 2004.

[7] ITU, Recommendation H.264, March 2005.

[8] Radha H.M., van der Schaar M. and Chen Y., "The mpeg-4 finegrained scalable video coding method for multimedia streaming over ip," Transactions on Multimedia, vol. 3, no. 1, pp. 53-68, March 2001.

[9] S. Savage, A. Collins, and E. Hoffman, "The end-to-end effects of internet path selection," in Proceedings of ACM SIGCOMM, 1999.

[10] J. Apostolopoulos, T. Wong, W. Tan, and S. Wee, "On multiple description streaming with content delivery networks," in Proceedings of IEEE INFOCOM, vol. 3, 23-27 June 2002, pp. 1736-1745.

[11] http://www.icir.org/models/tools.html.

[12] D. Jurca, S. Petrovic, and P. Frossard, "Media aware routing in large scale networks with overlay," in Proceedings of IEEE ICME, July 2005.

[13] Y. J. Liang, J. G. Apostolopoulos, and B. Girod, "Analysis of packet loss for compressed video: Does burst-length matter?" in Proceedings of IEEE ICASSP, 2003.

[14] K. Stuhlmuller, N. Farber, M. Link, and B. Girod, "Analysis of video transmission over lossy channels," IEEE Journal on Selected Areas in Communications, vol. 18, no. 6, pp. 1012-1032, June 2000.

[15] D. Jurca and P. Frossard, "Media-specific rate allocation in multipath overlay networks," EPFL," TR-ITS-2005.032, July 2005.

[16] Z. Michalewicz and D. B. Fogel, How to Solve It: Modern Heuristics, 2nd ed. Springer-Verlag, 2000.
[17] S. Vutukury and J. J. Garcia-Luna-Aceves, "Mdva: A distance-vector multipath routing protocol," in Proceedings of IEEE INFOCOM, 2001.

[18] W. Wei and A. Zakhor, "Multipath unicast and multicast video communication over wireless ad hoc networks," in Proceedings of IEEE/ACM BroadNets, October 2004

[19] D. Xu, Y. Chang, Y. Xiong, C. Qiao, and X. He, "On finding disjoint paths in single and dual link cost networks," in Proceedings of IEEE INFOCOM, March 2004.

[20] Y. Lee, Y. Seok, and Y. Choi, "Traffic engineering with constrained multipath routing in mpls networks," IEICE Transactions on Communications, vol. E85-A, no. 1, January 2002.

[21] F. Kelly and T. Voice, "Stability of end-to-end algorithms for joint routing and rate control," ACM SIGCOMM Computer Communcation Review, vol. 35, no. 2, pp. 5-12, April 2005.

[22] S. Tao and R. Guerin, "Application-specific path switching: A case study for streaming video," in Proceedings of ACM Multimedia, October 2004

[23] Z. Wang and J. Crowcroft, "Quality-of-service routing for supporting multimedia applications," IEEE Journal on Selected Areas in Communications, vol. 14, no. 7, pp. 1228-1234, September 1996.

[24] Y. Cui, K. Xu, and J. Wu, "Precomputation for multi-constrained qos routing in high-speed networks," in Proceedings of IEEE INFOCOM, 2003.

[25] T. Korkmaz and M. M. Krunz, "Routing multimedia traffic with qos guarantees," IEEE Transactions on Multimedia, vol. 5, no. 3, pp. 429443, September 2003.

[26] Z. Ma, H.-R. Shao, and C. Shen, "A new multi-path selection scheme for video streaming on overlay networks," in Proceedings of IEEE ICC, 2004.

[27] W.-L. Yang, "Optimal and heuristic algorithms for quality-of-service routing with multiple constraints," ACM Performance Evaluation, vol. 57, no. 3, pp. 261-278, July 2004.

[28] A. Valera, W. K. G. Seah, and S. V. Rao, "Cooperative packet caching and shortest multipath routing in mobile ad hoc networks," in Proceedings of IEEE INFOCOM, July 2003.

[29] J. Chestefield, R. Chakravorty, I. Pratt, S. Banerjee, and P. Rodriguez, "Exploiting diversity to enhance multimedia streaming over cellular links," in Proceedings of IEEE INFOCOM, March 2005.

[30] V. Srinivasan, C.-F. Chiasserini, P. S. Nugehalli, and R. R. Rao, "Optimal rate allocation for energy-efficient multipath routing in wireless ad hoc networks," IEEE Transactions on Wireless Communications, vol. 3, no. 3, pp. 891-899, May 2004

[31] K. C. Leung and V. O. K. Li, "Flow assignement and packet scheduling for multipath routing," Journal of Communications and Networks, vol. 5, no. 3, September 2003.

[32] J. Chen, S.-H. G. Chan, and V. O. K. Li, "Multipath routing for video delivery over bandwidth-limited networks," IEEE Journal on Selected Areas in Communications, vol. 22, no. 10, pp. 1920-1932, December 2004.

[33] T. Nguyen and A. Zakhor, "Path diversity with forward error correction (pdf) system for packet switched networks," in Proceedings of IEEE INFOCOM, 2003

[34] S. Mao, S. Lin, S. S. Panwar, Y. Wang, and E. Celebi, "Video transport over ad hoc networks: Multistream coding with multipath transport," IEEE Journal on Selected Areas in Communications, vol. 21, no. 10, pp. 1721-1737, December 2003.

[35] A. C. Begen, Y. Altunbasak, O. Ergun, and M. H. Ammar, "Multipath selection for multiple description video streaming over overlay networks," Signal Processing: Image Communcation, vol. 20, pp. 39-60, 2005 . 